\title{
No return to convexity
}

\author{
by \\ JAKub OnUfRY WojtaszczyK (Warszawa)
}

\begin{abstract}
We study the closures of classes of log-concave measures under taking weak limits, linear transformations and tensor products. We investigate which uniform measures on convex bodies can be obtained starting from some class $\mathcal{K}$. In particular we prove that if one starts from one-dimensional log-concave measures, one obtains no non-trivial uniform mesures on convex bodies.
\end{abstract}

1. Introduction and notation. The developments in asymptotic convex geometry generally tend to abandon the study of uniform measures on convex bodies in favour of log-concave measures. On one hand, this is a natural generalization - it is well-known that any log-concave measure is a weak limit of projections of uniform measures on convex bodies. On the other hand, it allows one to use a wide variety of tools hitherto unavailable. It also gives rise to a plethora of new examples (to give one: it is now possible to have a non-trivial one-dimensional case, as there are various one-dimensional logconcave measures, while one-dimensional convex bodies were a rather trivial object of study). A large number of strong results concerning convex bodies have been proved by passing through the domain of log-concave measures in a suitable way (see e.g. [7]).

A natural way to proceed in quite a few cases when considering the log-concave measures is some sort of induction upon dimension. As there is a well-developed theory of independent log-concave random variables, it is easy to study tensor products of such measures. It also frequently turns out that properties being considered are easily seen to be preserved under linear transformations and weak limits. One can give a number of examples of classes of measures closed under these three operations. If one restricts oneself to non-degenerate linear transformations, then measures with the isotropic constant $L_{\mu}$ bounded by some given $C$ form probably the most important class (see, for instance, [5] or [9] for an analysis of the isotropic

2010 Mathematics Subject Classification: Primary 52A23.

Key words and phrases: log-concave measure, product measure, weak limit, linear map, closure. 
constant problem). A more interesting class of transformations, where one is also allowed to use projections (and, generally, degenerate linear maps), preserves the infimum convolution inequality (see [8]), and the same set of operations was considered in [2]; a number of other examples are available.

In this paper we shall study the more general case (where arbitrary linear transformations are taken); of course the case of non-degenerate linear maps is contained in it. While in the case of uniform measures on convex bodies it is easy to see that not much new is going to be obtained by applying these operations (for instance, if we begin by taking the one-dimensional convex bodies, i.e. intervals, we end up only with parallelotopes), it is not obvious whether passing through the log-concave measures will help. One can see, for instance, that even starting only with intervals (that is, uniform measures on intervals), but working in the class of log-concave measures, one will obtain a wider variety of one-dimensional log-concave measures - for instance the gaussian measure as the limit case of projecting the uniform measure on the cube onto the line spanned by the vector $(1, \ldots, 1)$. Thus one might be inclined to hope that by proving that a property being studied is preserved under the given elementary operations in the log-concave setting one will obtain new, non-trivial examples of convex bodies satisfying the property. In this paper I intend to show that this is basically not the case. For instance, starting with all one-dimensional log-concave measures, one ends up with quite a number of log-concave measure (including non-product ones), but the only measures equidistributed on convex bodies one obtains are those equidistributed on parallelotopes.

The result is essentially negative - it proves that this is not the direction to pursue when attempting to prove new properties for convex bodies via log-concave measures. Its value, as in the case of most negative results, lies mainly in guiding other mathematicians away from this approach, rather than in direct application. As the approach, however, is not obviously wrong, the result still seems valuable.

1.1. Definitions and notation. The Lebesgue measure will be denoted by $\lambda$. For two sets $A, B$ in $\mathbb{R}^{n}$ we denote by $A+B$ their Minkowski sum, i.e. $\{a+b: a \in A, b \in B\}$, while $t A$ for a real number $t$ denotes $\{t a: a \in A\}$. A log-concave measure in $\mathbb{R}^{n}$ (where log-concave is short for logarithmically concave) is a measure satisfying $\mu(t A+(1-t) B) \geq \mu^{t}(A) \mu^{1-t}(B)$ for any $A, B \in \mathbb{R}^{n}$ and any $t \in(0,1)$. We will assume all our log-concave measures are probability measures and are not concentrated on any lower-dimensional subspace (that is, if $H \subset \mathbb{R}^{n}$ is an affine subspace of lower dimension, then $\mu(H)=0$ ). A celebrated result of Borell (see [1]) states that any log-concave measure satisfying the above conditions has a density $g$ with respect to the Lebesgue measure and $\log g$ is concave. 
We say a probability measure $\mu$ is isotropic if $\int_{\mathbb{R}^{n}} x d \mu(x)=0$ and $\int_{\mathbb{R}^{n}}\langle x, t\rangle^{2} d \mu(x)=1$ for any $t$ on the unit Euclidean sphere. It is easy to see that any measure not concentrated on a lower-dimensional subspace has an affine image which is isotropic.

We shall call a random variable isotropic, log-concave, etc. if it is distributed according to a law which is isotropic, log-concave, etc.

We say a class of measures is closed under products if for any $\mu_{1}, \mu_{2} \in \mathcal{K}$ we have $\mu_{1} \otimes \mu_{2} \in \mathcal{K}$. We say $\mathcal{K}$ is closed under linear transformations if for any linear map $T$ and any $\mu \in \mathcal{K}$ we have $\mu \circ T^{-1} \in \mathcal{K}$. In particular, by the projection of a measure $\mu$ we mean $\mu \circ P^{-1}$, where $P$ is a projection (in other contexts this is also referred to as a marginal of $\mu$ ). The projection of a log-concave measure is log-concave (this follows from the Prekopa-Leindler inequality, see e.g. (4)). Finally, we say $\mathcal{K}$ is closed under weak limits if for any sequence $\left(\mu_{n}\right)$ of measures from $\mathcal{K}$, if the weak limit $\mu$ of $\left(\mu_{n}\right)$ exists, then it belongs to $\mathcal{K}$.

$c, C, c_{1}, c_{2}, \ldots$ will always denote universal constants, possibly different from line to line. $c(n)$ and $C(n)$ are constants dependent only on the dimension $n$.

2. Tensorization does not help. This section is devoted to the proof of Theorem 2.15. which basically states that when one considers the closure of a class of measures with respect to products, linear transformations and weak limits and requires the result to be a uniform measure on a convex body, then it is enough to perform these operations one-by-one - the result is a product of weak limits of linear transformations.

2.1. Log-concave preliminaries. Recall we assume our measures to be probability measures. In this setting the following facts are well-known:

FACT 2.1. Let $\mu$ be an isotropic log-concave measure in $\mathbb{R}^{n}$. Then $\mu$ has a density $g$, and there exist constants $c(n), C(n)$, dependent on $n$, such that

$$
c(n) \leq g(0) \leq \sup _{x \in \mathbb{R}^{n}} g(x) \leq C(n) .
$$

The bound on $\sup g(x)$ is easily equivalent to the existence of a bound on the isotropic constant (precisely, $\sup g(x)^{1 / n} \simeq L_{K}$, where $\simeq$ denotes equality up to a universal constant), which may easily be seen to be bounded from above by $\sqrt{n}$; see [5] or [9] for information on this relation and [7] for the best currently known bound of $O(\sqrt[4]{n})$. The relation between $g(0)$ and $\sup g(x)$ is studied for instance by Matthieu Fradelizi in [3]. The lower bound is $c^{n}$ (see e.g. [9]).

FACT 2.2. Let $\mu$ be an isotropic log-concave measure in $\mathbb{R}^{n}$, let $g$ be its density and let $H$ be any central hyperplane. Then $g$ restricted to $H$ is a 
log-concave function, and there exist constants $c, C$ such that $c \leq \int_{H} g \leq C$ and for any $\theta \in H$ with $\|\theta\|_{2}=1$ we have $c \leq \int_{H}\langle x, \theta\rangle^{2} g(x) d x \leq C$.

Proof. For the first part, project $\mu$ onto $H^{\perp}$. We obtain a 1-dimensional isotropic $\log$-concave measure $\nu_{1}$, and the integral $\int_{H} d \mu$ is equal to the density of $\nu_{1}$ at zero, which is bounded from above and below by Fact 2.1 .

For the second fact, we project $\mu$ onto the plane spanned by $H^{\mathcal{L}}$ and $\theta$; the result is a 2-dimensional isotropic log-concave measure $\nu_{2}$. Let $\mathcal{L}$ be the line spanned by $\theta$ and let $h$ be the density of $\nu_{2}$. We have $C>\int_{\mathcal{L}} h(x) d x>c$ as above, and $C>\sup h(x) \geq h(0)>c$ by Fact 2.1. Define

$$
k=\sqrt{\int_{\mathcal{L}} x^{2} h(x) d x / \int_{\mathcal{L}} h(y) d y} .
$$

Notice that $\tilde{h}(x)=k h(k x) / \int_{\mathcal{L}} h(y) d y$ is log-concave; direct integration also proves that $\int_{\mathcal{L}} \tilde{h}(x) d x=1, \int_{\mathcal{L}} x \tilde{h}(x)=0$ and $\int_{\mathcal{L}} x^{2} \tilde{h}(x) d x=1$, thus $\tilde{h}$ is a density of a log-concave isotropic measure.

Now

$$
\int\langle\theta, x\rangle^{2} d \mu=\int_{\mathcal{L}} x^{2} h(x) d x=k^{2} \int_{\mathcal{L}} h(y) d y=\frac{\tilde{h}^{2}(0)\left(\int_{\mathcal{L}} h(y) d y\right)^{3}}{h^{2}(0)},
$$

where we use the definition of $\mathcal{L}$, then the definition of $k$ and finally the definition of $\tilde{h}$. As $\tilde{h}$ is isotropic on $\mathcal{L}$, we can bound $\tilde{h}(0)$ by universal constants from above and below; combined with the bounds for $h(0)$ and $\int_{\mathcal{L}} h(y) d y$ obtained before, this gives us the conclusion.

Note that we applied Fact 2.1 only to measures on $\mathbb{R}$ or $\mathbb{R}^{2}$, and not on $\mathbb{R}^{n}$ - thus the constants we got are indeed universal (dependent on the values $c(1), c(2)$, but not $c(n))$.

\subsection{Symmetrizations}

Definition 2.3. Let $f$ be any bounded measurable function on $\mathbb{R}$. Then the symmetrization of $f$ is the unique symmetric upper-continuous function $\tilde{f}$ which is decreasing on $\mathbb{R}_{+}$and satisfies $\lambda\{x: \tilde{f}(x) \geq c\}=\lambda\{x: f(x) \geq c\}$ for any $c$. If $f$ is a bounded measurable function on $\mathbb{R}^{n}$, and $\theta$ is a direction in $\mathbb{R}^{n}$, then the symmetrization of $f$ in the direction $\theta$ is a function $\tilde{f}$ such that for any $v \in \theta^{\perp}$ the function $\tilde{f}$ restricted to $v+\theta \mathbb{R}$ is the symmetrization of $f$ restricted to $v+\theta \mathbb{R}$.

Formally the definition above is correct only for $v$ s for which $f_{\mid v+\theta \mathbb{R}}$ is measurable, and for a set of $v$ s of measure 0 the restrictions may be nonmeasurable; however, we will apply this procedure to log-concave functions, for which the definition is correct everywhere.

Proposition 2.4. The symmetrization of a log-concave function is logconcave. 
Proof. Obviously for any bounded $f$ the symmetrization of $\log f$ is the logarithm of the symmetrization of $f$, thus it is enough to check that the symmetrization of a concave function is concave. Take any two points $x+r \theta$ and $y+s \theta$ with $x, y \in \theta^{\perp}$, and let $\tilde{f}(x+r \theta)=a$ and $\tilde{f}(y+s \theta)=b$. This means that $\lambda\{u: f(x+u \theta) \geq a\}=\lambda\{u: \tilde{f}(x+u \theta) \geq a\} \geq 2 r$. As $f$ is concave, $I:=\{u: f(x+u \theta) \geq a\}$ is an interval of length at least $2 r$, and similarly $J:=\{u: f(y+u \theta) \geq b\}$ is an interval of length at least $2 s$. Take any $t \in[0,1]$; then for $v \in t I+(1-t) J$ we have (by the concavity of $f) f(v) \geq t a+(1-t) b$. Moreover $t I+(1-t) J$ is an interval of $t x+(1-t) y+\theta \mathbb{R}$ of length at least $2(t r+(1-t) s)$. Thus $\lambda\{u: f(t x+(1-t) y+u \theta) \geq t a+(1-t) b\} \geq 2(t r+(1-t) s)$, and so $\tilde{f}(t(x+r \theta)+(1-t)(y+s \theta)) \geq t a+(1-t) b$, which (as $x, y, r, s$ and $t$ were arbitrary) proves the concavity of $\tilde{f}$.

Proposition 2.5. Let $\mu$ be a log-concave measure in $\mathbb{R}^{n}$ with density $g$, mean zero and a diagonal covariance matrix. Let $\tilde{g}$ be the symmetrization of $g$ in one of the coordinate directions $e_{i}$, and $\tilde{\mu}$ be the measure with density $\tilde{g}$. Then $\tilde{\mu}$ also has a diagonal covariance matrix, with $\mathbb{E}_{\mu}\left\langle X, e_{j}\right\rangle^{2}=\mathbb{E}_{\tilde{\mu}}\left\langle X, e_{j}\right\rangle^{2}$ for $j \neq i$. Moreover there exists a constant $c(n)>0$ dependent only on dimension such that $\mathbb{E}_{\mu}\left\langle X, e_{i}\right\rangle^{2} \geq \mathbb{E}_{\tilde{\mu}}\left\langle X, e_{i}\right\rangle^{2} \geq c(n) \mathbb{E}_{\mu}\left\langle X, e_{i}\right\rangle^{2}$.

Proof. First notice that the joint distribution of all $\left\langle X, e_{j}\right\rangle$ for $j \neq i$ is the same for $X$ distributed according to $\mu$ and to $\tilde{\mu}$, as the projections of these two measures onto $\operatorname{span}\left\{e_{j}: j \neq i\right\}$ are the same-thus all but the $i$ th row and $i$ th column of the respective covariance matrices are the same. Furthermore as $\tilde{g}$ is symmetric with respect to the hyperplane $\operatorname{span}\left\{e_{j}: j \neq i\right\}$, we have $\mathbb{E}_{\tilde{\mu}}\left\langle X, e_{i}\right\rangle\left\langle X, e_{j}\right\rangle=0$ for $i \neq j$. Also $\mathbb{E}_{\tilde{\mu}}\left\langle X, e_{i}\right\rangle^{2}=\int_{e_{i}^{\perp}} \int_{\mathbb{R}} x^{2} \tilde{g}\left(v+x e_{i}\right) d x d v \leq \int_{e_{i}^{\perp}} \int_{\mathbb{R}} x^{2} g\left(v+x e_{i}\right) d x d v$ by the monotone rearrangement inequality.

Consider the diagonal map $T_{\mu}$ (resp. $T_{\tilde{\mu}}$ ) transforming the measure $\mu$ to an isotropic measure. The $i$ th entry on the diagonal of the matrix of $T_{\mu}$ is equal to $1 / \sqrt{a_{i i}}$, where $a_{i i}$ (resp. $\tilde{a}_{i i}$ ) is the $i$ th diagonal entry of the covariance matrix of $\mu$. Let $M_{\mu}$ (resp. $M_{\tilde{\mu}}$ ) denote the supremum of the density of $\mu \circ T_{\mu}^{-1}$ (resp. $\tilde{\mu} \circ T_{\tilde{\mu}}^{-1}$ ), and let $M$ denote the common supremum of the densities of $\mu$ and $\tilde{\mu}$. We have $M_{\mu}=M / \operatorname{det} T_{\mu}$ and $M_{\tilde{\mu}}=M / \operatorname{det} T_{\tilde{\mu}}$, so

$$
M_{\tilde{\mu}} / M_{\mu}=\operatorname{det} T_{\mu} / \operatorname{det} T_{\tilde{\mu}}=\sqrt{\tilde{a}_{i i}} / \sqrt{a_{i i}}=\sqrt{\mathbb{E}_{\tilde{\mu}}\left\langle X, e_{i}\right\rangle^{2} / \mathbb{E}_{\mu}\left\langle X, e_{i}\right\rangle^{2}},
$$

where the middle equality follows as all eigenvalues of $\operatorname{cov} \mu$ and $\operatorname{cov} \tilde{\mu}$ except the $i$ th are equal.

On the other hand both $\mu \circ T_{\mu}$ and $\tilde{\mu} \circ T_{\tilde{\mu}}$ are isotropic log-concave measures, thus by Fact 2.1 we have $M_{\tilde{\mu}} / M_{\mu} \geq c(n) / C(n)$. This ends the proof. 
Proposition 2.6. Let $\mu$ be a log-concave probability measure with density $g$, mean zero and a diagonal covariance matrix. For $z \in e_{i}^{\perp}$ let $g^{\prime}(z)=$ $\sup _{x \in \mathbb{R}} g\left(z+r e_{i}\right)$. Then:

- $g^{\prime}(z)$ is a log-concave function on $e_{i}^{\perp}$;

- $c(n) / \sqrt{\mathbb{E}\left\langle X, e_{i}\right\rangle^{2}} \leq \int_{e_{i}^{\perp}} g^{\prime}(z) \leq C(n) / \sqrt{\mathbb{E}\left\langle X, e_{i}\right\rangle^{2}}$.

Proof. Let $\tilde{g}$ be the symmetrization of $g$ in direction $e_{i}$. Then $\tilde{g}(z, 0)=$ $g^{\prime}(z)$-both are equal to $\sup _{r \in \mathbb{R}} g\left(z+r e_{i}\right)$. Thus by Proposition 2.4 $g^{\prime}$ is log-concave. By Proposition 2.5 the measure with density $\tilde{g}$ has a diagonal covariance matrix; as before consider a diagonal map $T$ which transforms this measure into an isotropic one, let $h$ be the density of this isotropic measure. By Fact 2.2 we have $c(n) \int_{e_{i}^{\perp}} h \leq C(n)$. Now the $i$ th diagonal entry of $T$ is $\sqrt{\mathbb{E}\left\langle X, e_{i}\right\rangle^{2}}$, so this is the factor by which the mass on the hyperplane $e_{i}^{\perp}$ is changed by $T$.

\subsection{The Lipschitz invariant}

Proposition 2.7. Let $X_{1}$ and $X_{2}$ be two independent random variables in $\mathbb{R}^{n}$ satisfying

$$
\forall_{\theta:\|\theta\|_{2}=1} \quad c<\mathbb{E}\left\langle X_{i}, \theta\right\rangle^{2}<C
$$

for some positive constants $c, C$. Let $W$ and $V$ be two diagonal matrices with $W^{2}+V^{2}=$ Id. Then $c<\mathbb{E}\left\langle W X_{1}+V X_{2}, \theta\right\rangle^{2}<C$.

Proof. Let $G_{1}$ and $G_{2}$ denote the covariance matrices of $X_{1}$ and $X_{2}$, respectively. The assumption on $\mathbb{E}\left\langle X_{i}, \theta\right\rangle^{2}$ simply means that all the eigenvalues of $G_{1}$ and $G_{2}$ lie in the interval $[c, C]$.

We have $\operatorname{cov}\left(W X_{1}+V X_{2}\right)=\operatorname{cov}\left(W X_{1}\right)+\operatorname{cov}\left(V X_{2}\right)=W G_{1} W+V G_{2} V$. Take any vector $\theta$ of norm 1 . Then $\left\langle W G_{1} W \theta, \theta\right\rangle=\left\langle G_{1} W \theta, W \theta\right\rangle$. As all the eigenvalues of $G_{1}$ are no smaller than $c$, we have $\left\langle G_{1} v, v\right\rangle \geq c\|v\|^{2}$ for any $v$, thus $\left\langle W G_{1} W \theta, \theta\right\rangle \geq c\|W \theta\|^{2}$. Similarly $\left\langle V G_{2} V \theta, \theta\right\rangle \geq c\|V \theta\|^{2}$. As $V$ and $W$ are diagonal, we have $\|W \theta\|^{2}=\sum w_{i i}^{2} \theta_{i}^{2}$, and as $W^{2}+V^{2}=\mathrm{Id}$, we have $\|W \theta\|^{2}+\|V \theta\|^{2}=\sum\left(w_{i i}^{2}+v_{i i}^{2}\right) \theta_{i}^{2}=\sum \theta_{i}^{2}=\|\theta\|^{2}=1$. Thus

$$
\left\|\operatorname{cov}\left(W X_{1}+V X_{2}\right) \theta\right\| \geq\left\langle\operatorname{cov}\left(W X_{1}+V X_{2}\right) \theta, \theta\right\rangle \geq c,
$$

so in particular all the eigenvalues of $\operatorname{cov}\left(W X_{1}+V X_{2}\right)$ must be greater than or equal to $c$ (they are all positive reals, as eigenvalues of a covariance matrix). A similar argument gives the upper bound on the eigenvalues.

Lemma 2.8. Let $g$ and $h$ be the densities of two independent log-concave random variables $X$ and $Y$ in $\mathbb{R}^{n}$ with diagonal covariance matrices satisfying $\operatorname{cov}(X+Y)=$ Id. Let $f^{\prime}(v)=\sup _{t \in \mathbb{R}} f\left(v+t e_{i}\right)$ for $f \in\{g, h\}$ and $v \in e_{i}^{\perp}$. Then 


$$
\int_{e_{i}^{\perp}} g^{\prime}(v) h^{\prime}(z-v) d v \leq C(n) / \sqrt{\mathbb{E}\left\langle X, e_{i}\right\rangle^{2} \mathbb{E}\left\langle Y, e_{i}\right\rangle^{2}}
$$

for some constant $C(n)$ dependent only on $n$.

Proof. Begin by considering the symmetrization $g_{1}$ of $g$ with respect to $e_{i}$. Let this be the density of a variable $\tilde{X}$. By Proposition 2.5, $\tilde{X}$ has a diagonal covariance matrix $G$, and $G^{-1 / 2} \tilde{X}$ is isotropic.

Let $g_{2}$ be the density of $G^{-1 / 2} \tilde{X}$. Consider the restriction of $g_{2}$ to $e_{i}^{\perp}$. Let $c_{2}:=\int_{e_{i}^{\perp}} g_{2}$ and $g_{3}:=g_{2} / c_{2}$. Then $g_{3}$ is the density of a probability measure on $e_{i}^{\perp}$, which is also obviously log-concave. Let $X_{3}$ be a random variable distributed according to $g_{3}$. By Fact 2.2 the eigenvalues of cov $X_{3}$ are in some universal interval $[c, C]$ and $c<c_{2}<C$.

Let $g_{4}$ be the restriction of $g_{1}$ to $e_{i}^{\perp}$, and $c_{4}:=\int_{e_{i}^{\perp}} g_{4}$ and $g_{5}:=g_{4} / c_{4}$; finally let $X_{5}$ be a random variable distributed according to $g_{5}$. Note that $g^{\prime}$ is equal to $g_{4}$. We define $h_{2}, d_{2}, h_{3}, Y_{3}, h_{4}, d_{4}, h_{5}$ and $Y_{5}$ analogously to $g_{2}, c_{2}, g_{3}, X_{3}, g_{4}, c_{4}, g_{5}$ and $X_{5}$, only starting from $h$ being the density of $Y$.

By the construction above and by Proposition 2.5 we have

$$
c_{2} / c_{4}=\sqrt{G_{i i}} \geq c(n) \sqrt{(\operatorname{cov} X)_{i i}} .
$$

Also let $G^{\prime}$ be the restriction of $G$ to $e_{i}^{\perp}$ (that is, the matrix obtained from $G$ by deleting the $i$ th row and $i$ th column). Then $X_{5}=G^{1 / 2} X_{3}$. We perform similar operations on $h$ to obtain

$$
d_{2} / d_{4} \geq c(n) \sqrt{(\operatorname{cov} Y)_{i i}}
$$

$Y_{5}=H^{1 / 2} Y_{3}$ and the eigenvalues of cov $Y_{3}$ are in the same interval $[c, C]$.

We are now in the situation of Proposition 2.7. We have random variables $X_{3}$ and $Y_{3}$, satisfying $c<\mathbb{E}\left\langle X_{3}, \theta\right\rangle^{2}<C$ for $\|\theta\|=1$, and the same for $Y_{3}$, and matrices $G^{\prime 1 / 2}$ and $H^{\prime 1 / 2}$, whose squares sum up to the identity matrix by Proposition 2.5 and the assumption $\operatorname{cov}(X+Y)=\mathrm{Id}$. Thus the variable $X_{5}+Y_{5}$ satisfies the conclusion of Proposition 2.7, that is, all the eigenvalues of its covariance matrix lie in the interval $[c, C]$.

The integral we consider, $\int_{e_{i}^{\perp}} g^{\prime}(v) h^{\prime}(z-v) d v$, is equal to $d_{4} c_{4}$ times the density of the variable $X_{5}+Y_{5}$ at $z$. Recall that $c_{2}$ and $d_{2}$ are universally bounded by Fact 2.2. Thus by 2.1 and $(2.2$ we only have to prove that the density of $X_{5}+Y_{5}$ is bounded by a constant dependent on $n$.

Let $M$ be a diagonal matrix such that $M\left(X_{5}+Y_{5}\right)$ is isotropic, that is, $M=\operatorname{cov}\left(X_{5}+Y_{5}\right)^{-1 / 2}$. Then the density of $M\left(X_{5}+Y_{5}\right)$ is bounded from above by $C(n)$ by Fact 2.1, and the supremum of the density of $X_{5}+Y_{5}$ is equal to the supremum of the density of $M\left(X_{5}+Y_{5}\right)$ multiplied by $\operatorname{det} M=$ $\operatorname{det} \operatorname{cov}\left(X_{5}+Y_{5}\right)^{-1 / 2} \leq c^{-n / 2}$ for some $c>0$ (as all the eigenvalues of $\operatorname{cov}\left(X_{5}+Y_{5}\right)$ are bounded from below by universal positive constants).

And now for the final result of this section: 
Definition 2.9. We say a function $f: \mathbb{R}^{n} \rightarrow \mathbb{R}$ is Lipschitz in direction $\theta$ (where we assume $\|\theta\|_{2}=1$ ) with a constant $L$ if for any $x \in \mathbb{R}^{n}$ and $t \in \mathbb{R}$ we have $|f(x+t \theta)-f(x)| \leq L|t|$.

Proposition 2.10. Let $X$ and $Y$ be two independent log-concave random variables in $\mathbb{R}^{n}$ with diagonal covariance matrices such that $X+Y$ is isotropic. If $\mathbb{E}\left\langle X, e_{i}\right\rangle^{2}$ and $\mathbb{E}\left\langle Y, e_{i}\right\rangle^{2}$ are both positive, then the density of $X+Y$ is Lipschitz in direction $e_{i}$ with the Lipschitz constant bounded by $C(n) / \sqrt{\mathbb{E}\left\langle X, e_{i}\right\rangle^{2} \mathbb{E}\left\langle Y, e_{i}\right\rangle^{2}}$.

Proof. We may assume $X$ and $Y$ have densities $g$ and $h$ respectively by convoluting each with a gaussian variable $\varepsilon G$ (where $G \sim \mathcal{N}(0$, Id)), as the density of $X+Y+\varepsilon G+\varepsilon G$ tends to the density of $X+Y$ when $\varepsilon \rightarrow 0$, and the Lipschitz constant is preserved under pointwise convergence.

The density $f$ of $X+Y$ is the convolution of $g$ and $h$. For any $v \in \mathbb{R}^{n}$ we denote its decomposition into $e_{i}^{\perp}$ and $\operatorname{span}\left\{e_{i}\right\}$ by $v=\tilde{v}+t_{v} e_{i}$. We have

$$
f(x)=\int_{\mathbb{R}^{n}} g(y) h(x-y) d y=\iint_{e_{i}^{\perp}} \int_{\mathbb{R}} g\left(\tilde{y}+t_{y} e_{i}\right) h\left(\tilde{x}-\tilde{y}+\left(t_{x}-t_{y}\right) e_{i}\right) d t_{y} d \tilde{y},
$$

and so the difference $\left|f\left(x+s e_{i}\right)-f(x)\right|$ is equal to

$$
\left|\iint_{e_{i}^{\perp}} g\left(\tilde{\mathbb{y}}+t e_{i}\right)\left[h\left(\tilde{x}-\tilde{y}+\left(t_{x}+s-t_{y}\right) e_{i}\right)-h\left(\tilde{x}-\tilde{y}+\left(t_{x}-t_{y}\right) e_{i}\right)\right] d t_{y} d \tilde{y}\right| .
$$

Let $g^{\prime}(\tilde{v})=\sup _{t \in \mathbb{R}} g\left(\tilde{v}+t e_{i}\right)$ and $h^{\prime}(\tilde{v})=\sup _{t \in \mathbb{R}}\left(\tilde{v}+t e_{i}\right)$. Then we have

$$
\begin{aligned}
& \left|f\left(x+s e_{i}\right)-f(x)\right| \\
& \quad \leq \int_{e_{i}^{\perp}} \int_{\mathbb{R}} g\left(\tilde{y}+t e_{i}\right)\left|h\left(\tilde{x}-\tilde{y}+\left(t_{x}+s-t_{y}\right) e_{i}\right)-h\left(\tilde{x}-\tilde{y}+\left(t_{x}-t_{y}\right) e_{i}\right)\right| d t_{y} d \tilde{y} \\
& \leq \int_{e_{i}^{\perp}} g^{\prime}(\tilde{y}) \int_{\mathbb{R}}\left|h\left(\tilde{x}-\tilde{y}+\left(t_{x}+s-t_{y}\right) e_{i}\right)-h\left(\tilde{x}-\tilde{y}+\left(t_{x}-t_{y}\right) e_{i}\right)\right| d t_{y} d \tilde{y} .
\end{aligned}
$$

Notice that $h\left(\tilde{v}+t e_{i}\right)$ is log-concave, and thus bimonotone, as a function of $t$. Thus the function $h\left(\tilde{v}+(t+s) e_{i}\right)-h\left(\tilde{v}+t e_{i}\right)$ for positive $s$ has a single zero at some $t_{0}$, is non-negative for $t<t_{0}$ and non-positive for $t>t_{0}$. Thus

$$
\begin{aligned}
\int_{\mathbb{R}} \mid h(\tilde{v} & \left.+(t+s) e_{i}\right)-h\left(\tilde{v}+t e_{i}\right) \mid d t \leq \int_{-\infty}^{t_{0}} h\left(\tilde{v}+(t+s) e_{i}\right)-h\left(\tilde{v}+t e_{i}\right) d t \\
& -\int_{t_{0}}^{\infty} h\left(\tilde{v}+(t+s) e_{i}\right)-h\left(\tilde{v}+t e_{i}\right) d t=2 \int_{t_{0}}^{t_{0}+s} h\left(\tilde{v}+t e_{i}\right) d t \leq 2|s| h^{\prime}(\tilde{v}) .
\end{aligned}
$$


Applying this to our case we have

$$
\left|f\left(x+s e_{i}\right)-f(x)\right| \leq \int_{e_{i}^{\perp}} g^{\prime}(\tilde{y}) 2|s| h^{\prime}(\tilde{x}-\tilde{y}) d \tilde{y} .
$$

Here, however, we may apply Lemma 2.8 to conclude the proof.

2.4. Closed classes. We begin by demonstrating a simple structural proposition:

Proposition 2.11. Let $\mathcal{K}$ be a class of log-concave measures. Let $\overline{\mathcal{K}}$ be the smallest class of log-concave measures containing $\mathcal{K}$, which is closed under products, linear transformations and weak limits. Let $\mu$ be any isotropic measure in $\overline{\mathcal{K}}$. Then there exists a sequence of measures $\mu_{1}, \mu_{2}, \ldots$, each of which is a linear image of the tensor product of finitely many measures from $\mathcal{K}$, such that $\mu$ is the weak limit of $\mu_{i}$, and each $\mu_{i}$ is isotropic.

Proof. First let $\mathcal{L}$ be the smallest class of log-concave measures containing $\mathcal{K}$, which is closed under products and linear transformations. We shall prove that any member of $\overline{\mathcal{K}}$ is a weak limit of some sequence in $\mathcal{L}$. Let $\overline{\mathcal{L}}$ be the set of all weak limits of sequences in $\mathcal{L}$. We will prove that $\overline{\mathcal{L}}$ is closed under products, linear transformations and weak limits, thus $\overline{\mathcal{L}}=\overline{\mathcal{K}}$. Let $\mu_{1}, \mu_{2} \in \overline{\mathcal{L}}$, say $\mu_{1}=\lim \nu_{k}, \mu_{2}=\lim \omega_{k}$. Then $\mu_{1} \otimes \mu_{2}=\lim \nu_{k} \otimes \omega_{k}$. Similarly, if $T$ is a linear transformation, then $\left(\lim \nu_{k}\right) \circ T^{-1}=\lim \left(\nu_{k} \circ T^{-1}\right)$. Thus $\overline{\mathcal{L}}$ is closed under products and linear transformations. Now recall that the space of probability measures with the weak convergence can be viewed as a metric space with the Lévy metric. Thus the closure of any set with respect to weak limits is simply the closure in the Lévy metric, and this is precisely the set of all weak limits of sequences in this set. Thus $\overline{\mathcal{L}}=\overline{\mathcal{K}}$.

Let $\mu=\lim \mu_{k}$ with $\mu_{k} \in \mathcal{L}$. Let $\nu_{k}=\mu_{k} \circ\left(\operatorname{cov} \mu_{k}\right)^{-1 / 2}$. As $\mu$ is isotropic and the covariance matrix is continuous for log-concave measures, cov $\mu_{k} \rightarrow$ Id and thus also $\nu_{k} \rightarrow \mu$. The measures $\nu_{k}$ are isotropic.

Now we have to consider $\nu_{k}$ which are elements of $\mathcal{L}$. We prove each $\nu_{k}$ is the linear image of a product by structural induction, exchanging all linear transformations with products, as $\left(\mu \circ S^{-1}\right) \otimes\left(\nu \circ T^{-1}\right)=(\mu \otimes \nu) \circ(S \otimes T)^{-1}$.

LEMma 2.12. Let $\mu_{k}$ be a sequence of isotropic log-concave probability measures with densities $f_{k}$, weakly convergent to some $\mu$. Assume each $f_{k}$ is Lipschitz in some direction $v_{k}$ with the same constant L. Then $\mu$ has a density $f$ that is Lipschitz with constant $L$ in some direction $v$.

Proof. First note that $\mu$ has to be log-concave and isotropic, and thus has a density. Now pass to a subsequence of $k \mathrm{~s}$ such that $v_{k}$ is convergent to some $v$. 
Let $h_{\delta, z}(x)=\max \{1-|x-z| / \delta, 0\}$, and let

$$
T_{\delta, z}(\mu)=\int h_{\delta, z}(x) d \mu(x) / \int h_{\delta, z}(x) d x .
$$

For any $k$ the function $T_{\delta, z}\left(\mu_{k}\right)$ is $L$-Lipschitz in direction $v_{k}$ as a function of $z$. Fix a point $z$ and a constant $t>0$. We have

$$
\begin{aligned}
& \left|T_{\delta, z+t v_{k}}\left(\mu_{k}\right)-T_{\delta, z+t v}(\mu)\right| \\
& \quad \leq\left|T_{\delta, z+t v_{k}}\left(\mu_{k}\right)-T_{\delta, z+t v}\left(\mu_{k}\right)\right|+\left|T_{\delta, z+t v}\left(\mu_{k}\right)-T_{\delta, z+t v}(\mu)\right| .
\end{aligned}
$$

The first summand tends to zero as $h_{\delta, z+t v_{k}}$ tends to $h_{\delta, z+t v}$ uniformly, while the density of $\mu_{k}$ is bounded uniformly in $k$. The second summand tends to zero as $T$ is a continuous functional of a measure. Thus, in particular, $T_{\delta, z}(\mu)$ is an $L$-Lipschitz function of $z$. Note that the Lipschitz constant is independent of $\delta$.

We modify $f$ to be zero on the boundary of supp $\mu$-this is a modification on a set of measure 0 , so the modified $f$ is also a density of $\mu$. We will prove $f$ is now $L$-Lipschitz in the direction $v$. Take any point $z$ and consider the line $\mathcal{L}=z+t v$. If this line does not intersect the interior of $\operatorname{supp} \mu$, then $f$ is equal to 0 on $\mathcal{L}$, and thus is $L$-Lipschitz. Now suppose $\mathcal{L}$ intersects the interior of $\operatorname{supp} \mu$. As $\operatorname{supp} \mu$ is convex, $\mathcal{L}$ intersects the boundary of $\operatorname{supp} \mu$ in exactly two points. Take any two points $x, y$ on $\mathcal{L}$, different from the two intersection points. Then $f$ is continuous in some neighbourhoods of $x$ and $y$, for $f$ is continuous both in the interior of $\operatorname{supp} \mu$ and outside supp $\mu$. Thus $T_{\delta, x} \rightarrow f(x)$ and $T_{\delta, y} \rightarrow f(y)$ when $\delta \rightarrow 0$, so $f$ is Lipschitz everywhere except at the two boundary points (but also when $x, y$ straddle a boundary point). To deal with the case of $x$ or $y$ lying on the boundary of $\operatorname{supp} \mu$ we take a sequence $x_{k} \rightarrow x$ (or $y_{k} \rightarrow y$, respectively).

Lemma 2.13. Let $(n+1)^{-2}>\varepsilon>0$. Let $X_{1}, \ldots, X_{k}$ be a sequence of independent random variables in $\mathbb{R}^{n}$ such that $\sum_{i=1}^{k} X_{i}$ is isotropic. Let $\mathcal{M}_{i}$ denote the space spanned by the eigenvectors of $\operatorname{cov} X_{i}$ corresponding to eigenvalues larger than $1-\varepsilon$. Then either

- $\sum_{i} \operatorname{dim} \mathcal{M}_{i}=n$, or

- there exists a subset $S$ of $\{1, \ldots, k\}$ such that $\operatorname{cov} \sum_{i \in S} X_{i}$ has an eigenvalue $\lambda$ satisfying $\varepsilon<\lambda<1-\varepsilon$.

Proof. Let $A_{i}$ denote the covariance matrix of $X_{i}$. If any single $A_{i}$ has an eigenvalue between $\varepsilon$ and $1-\varepsilon$, we set $S=\{i\}$. Consider eigenvalues of $A_{i}$ s larger than $1-\varepsilon$; assume $A_{i}$ has $l_{i}$ such eigenvalues, and let $l=\sum_{i} l_{i}$. We have $l \leq n$, for otherwise $n=\operatorname{tr} \operatorname{Id}=\operatorname{tr} \sum A_{i} \geq \sum_{i} l_{i}(1-\varepsilon) \geq(n+1)(1-\varepsilon)>n$. If $l=d$ the first condition is satisfied. Otherwise there are at most $n-1$ indices $i$ with $l_{i}>0$, and the sum of the traces of the appropriate $A_{i} \mathrm{~s}$ is at most the sum of the large eigenvalues (at most $n-1$ ) and the sum of the 
small eigenvalues (at most $(n-1)^{2} \varepsilon$, for each of the matrices has at most $n-1$ small eigenvalues). Thus the trace of the sum of the remaining matrices is at least $1-(n-1)^{2} \varepsilon$.

Rearrange the vectors so that $l_{i}=0$ for $i=1, \ldots, m$, and $l_{i}>0$ for $i>m$. Consider the sums $b(j)=\sum_{i=1}^{j} \operatorname{tr} A_{i}$. We have $b(0)=0, b(m) \geq$ $1-(n-1)^{2} \varepsilon \geq 4 n \varepsilon$ and $b(j+1)-b(j) \leq n \varepsilon$. Thus for some $j_{0}$ we shall have $n \varepsilon \leq b\left(j_{0}\right) \leq 2 n \varepsilon$. We put $S=\left\{1, \ldots, j_{0}\right\}$. The vector $\sum_{i=1}^{j_{0}} X_{i}$ has to have an eigenvalue of the covariance matrix no smaller than $\varepsilon$ (for the trace of this matrix is at least $n \varepsilon$ ); on the other hand all its eigenvalues are no larger than the trace, which in turn is no larger than $2 n \varepsilon<1-\varepsilon$. Thus $S$ satisfies the theorem.

THEOREM 2.14. Let $Y_{k}=\sum_{i=1}^{i(k)} X_{k, i}$ be isotropic, log-concave random variables on $\mathbb{R}^{n}$. Assume all $X_{k, i}$ are independent. Assume $Y_{k} \rightarrow Y$ weakly, where $Y$ is a uniform measure on a convex body. Then $Y=Z_{1}+\cdots+Z_{m}$ for some independent random variables $Z_{j}$, where each $Z_{j}$ is a weak limit of some of the $X_{k, i}$ s, and all $Z_{j}$ are supported on orthogonal subspaces.

Proof. Choose any $\varepsilon>0$ and apply Lemma 2.13 for each $Y_{k}$. If for some $k$ the second case of the lemma occurs, we have $Y_{k}=Y_{k}^{\prime}+Y_{k}^{\prime \prime}$ (where $Y_{k}^{\prime}$ is the sum of the $X_{k, i} \mathrm{~s}$ for $i \in S$, and $Y_{k}^{\prime \prime}$ is the sum of the others), and some eigenvalue of $Y_{k}^{\prime}$ is between $\varepsilon$ and $1-\varepsilon$. As $Y_{k}$ is isotropic, $Y_{k}^{\prime \prime}$ has the same eigenvectors as $Y_{k}^{\prime}$, thus we may apply Proposition 2.10 to find that the density of $Y_{k}$ is $C(n) / \varepsilon$-Lipschitz in the direction of the appropriate eigenvector. If this occurs an infinite number of times, we may apply Lemma 2.12 to the subsequence of those $k \mathrm{~s}$ to conclude that the density of $Y$ is $C(n) / \varepsilon$-Lipschitz in some direction, and this contradicts the assumption that $Y$ was uniform on a convex body.

Thus for any $\varepsilon>0$ the second case of the lemma occurs only finitely many times. We may thus pass to a subsequence on which the first case occurs for each $Y_{k}$ with some $\varepsilon_{k}$ tending to zero. Thus for each $k$ we have a set of at most $d$ linear spaces $\mathcal{M}_{k, i}$. We pass to a subsequence again, so that the number and dimensions of the subspaces are constant, and again (after an appropriate rearrangement) to have $\mathcal{M}_{k, i} \rightarrow \mathcal{M}_{i}$ (the convergence of linear subspaces is taken, for instance, in the metric of the grassmannian manifold; this can be done due to the compactness of this manifold).

The spaces $\mathcal{M}_{i}$ have to be orthogonal. Assume, say, $\mathcal{M}_{1}$ and $\mathcal{M}_{2}$ are not orthogonal, say some two unit vectors $v_{1} \in \mathcal{M}_{1}$ and $v_{2} \in \mathcal{M}_{2}$ satisfy $\left\langle v_{1}, v_{2}\right\rangle=c<0$. Then we have sequences $v_{k, 1} \rightarrow v_{1}$ in $\mathcal{M}_{1}$ and $v_{k, 2} \rightarrow v_{2}$ in $\mathcal{M}_{2}$. Note the following inequalities:

$$
\begin{aligned}
1 & =\mathbb{E}\left\langle v_{k, 1}, Y_{k}\right\rangle^{2} \geq \mathbb{E}\left\langle v_{k, 1}, X_{k, 1}+X_{k, 2}\right\rangle^{2}=\mathbb{E}\left\langle v_{k, 1}, X_{k, 1}\right\rangle^{2}+\left\langle v_{k, 1}, X_{k, 2}\right\rangle^{2} \\
& \geq 1-\varepsilon_{k}+\mathbb{E}\left\langle v_{k, 1}, X_{k, 2}\right\rangle^{2},
\end{aligned}
$$


thus $\mathbb{E}\left\langle v_{k, 1}, X_{k, 2}\right\rangle^{2} \leq \varepsilon_{k}$. Moreover

$$
\begin{aligned}
\mathbb{E}\left\langle v_{k, 1}+v_{k, 2}, X_{k, 1}\right\rangle^{2} & =\mathbb{E}\left\langle v_{k, 1}, X_{k, 1}\right\rangle^{2}+2\left\langle v_{k, 1}, X_{k, 1}\right\rangle\left\langle v_{k, 2}, X_{k, 1}\right\rangle+\left\langle v_{k, 2}, X_{k, 1}\right\rangle^{2} \\
& \geq 1-\varepsilon_{k}-2\left|\mathbb{E}\left\langle v_{k, 1}, X_{k, 1}\right\rangle\left\langle v_{k, 2}, X_{k, 1}\right\rangle\right| \geq 1-\varepsilon_{k}-2 \sqrt{\varepsilon_{k}} .
\end{aligned}
$$

Finally

$$
\mathbb{E}\left\langle v_{k, 1}+v_{k, 2}, Y_{k}\right\rangle^{2} \geq \mathbb{E}\left\langle v_{k, 1}+v_{k, 2}, X_{k, 1}+X_{k, 2}\right\rangle^{2} \geq 2\left(1-\varepsilon_{k}-2 \sqrt{\varepsilon_{k}}\right),
$$

which is arbitrarily close to 2 for large enough $k$. On the other hand, however, $\left\langle v_{k, 1}+v_{k, 2}, v_{k, 1}+v_{k, 2}\right\rangle=2+2\left\langle v_{k, 1}, v_{k, 2}\right\rangle \rightarrow 2-2 c$, thus we should have $\mathbb{E}\left\langle v_{k, 1}+v_{k, 2}, Y_{k}\right\rangle^{2} \rightarrow 2-2 c$, a contradiction.

Now each $X_{k, i}$ for fixed $i$ converges weakly to some measure $Z_{i}$ distributed on $\mathcal{M}_{i}$ (for the variance in the directions orthogonal to $\mathcal{M}_{i}$ tends to zero, as shown above), and $Y$ is the sum of $Z_{i}$ s.

Now all that remains is to combine the theorem above with Proposition 2.11

Main Theorem 2.15. Let $\mathcal{K}$ be any class of log-concave measures closed under linear transformations, and let $\overline{\mathcal{K}}$ be the smallest class of log-concave measures containing $\mathcal{K}$ which is closed under products, weak limits and linear transformations. Let $\mu$ be any isotropic measure in $\overline{\mathcal{K}}$ which is a uniform measure on some convex body. Then $\mu$ is a product of some measures $\mu_{1}, \ldots, \mu_{n}$, each of which is a weak limit of some sequence of linear images of measures in $\mathcal{K}$.

2.5. Applications and discussion. Let us apply this result to a typical case.

COROLlary 2.16. Let $\mathcal{K}$ be the smallest class of log-concave measures, closed under products, linear transformations and weak limits, which contains all 1-dimensional log-concave measures. Let $\mu \in \mathcal{K}$ be the uniform measure distributed on a convex body $B$. Then $B$ is a parallelotope.

Proof. The class of 1-dimensional log-concave measures is closed under linear transformations and weak limits, thus by Theorem 2.15 any uniform measure on a convex body in $\mathcal{K}$ which is isotropic has to be a product of 1-dimensional log-concave measures, and thus equidistributed on the hypercube. Taking any, not necessarily isotropic, measure equidistributed on some $B$ in $\mathcal{K}$ we can take a linear transformation to make it isotropic, and as after this transformation $B$ is a hypercube, it had to be a parallelotope before.

Of course a case-by-case survey of what can be obtained from various classes of log-concave measures is impossible here. However the main scheme should be clear: we begin with some class of measures $\mathcal{K}$, and if there are no non-trivial convex bodies obtained as weak limits of linear transformations of measures from $\mathcal{K}$, then $\overline{\mathcal{K}}$ contains no non-trivial convex bodies. It would 
be interesting to prove, for instance, that the class of $\ell_{p}$ balls (for fixed $p$ between 1 and $\infty$ ) generates no non-trivial convex bodies (that is, convex bodies not being linear transforms of $\ell_{p}$ balls).

Acknowledgements. I would first and foremost like to thank the supervisor of my PhD thesis, Rafal Latała, for his unending patience and enormous help (in this case consisting both of presenting the question to me and answering my questions, especially in probability theory). I would like to thank Grigoris Paouris, who posed this question in July and motivated me to work on it. I would like to thank Radek Adamczak for pointing out an important mistake in my reasoning. I would also like to thank all the people who made my life somewhat easier in the last few months, in particular Łukasz and Iwona Degórski, Marcin and Olga Pilipczuk and Kasia Staniszewska. Finally I would like to thank Jakub Pochrybniak for his help with my technical $\mathrm{T}_{\mathrm{E}} \mathrm{X}$-related problems.

\section{References}

[1] C. Borell, Convex set functions in d-space, Period. Math. Hungar. 6 (1975), 111-136.

[2] N. Dafnis and G. Paouris, Small ball probability estimates, $\psi_{2}$ behavior and the hyperplane conjecture, J. Funct. Anal. 258 (2010), 1933-1964.

[3] M. Fradelizi, Sections of convex bodies through their centroid, Arch. Math. (Basel) 69 (1997), 515-522.

[4] R. J. Gardner, The Brunn-Minkowski inequality, Bull. Amer. Math. Soc. 39 (2002), 355-405.

[5] A. Giannopoulos, Notes on isotropic convex bodies, Warsaw Univ. Notes, 2003.

[6] D. Hensley, Slicing convex bodies-bounds for slice area in terms of the body's covariance, Proc. Amer. Math. Soc. 79 (1980), 619-625.

[7] B. Klartag, On convex perturbations with a bounded isotropic constant, Geom. Funct. Anal. 16 (2006), 1274-1290.

[8] R. Latała and J. O. Wojtaszczyk, On the infimum convolution inequality, Studia Math. 189 (2008), 147-187.

[9] V. D. Milman and A. Pajor, Isotropic positions and inertia ellipsoids and zonoids of the unit ball of a normed n-dimensional space, in: GAFA Seminar 87-89, Lecture Notes in Math. 1376, Springer, 1989, 64-104.

Jakub Onufry Wojtaszczyk

Institute of Mathematics

Polish Academy of Sciences

Śniadeckich 8

00-956 Warszawa, Poland

E-mail: onufry@mimuw.edu.pl 